"This is the peer reviewed version of the following article: [Regmi, P, Aryal, N, kurmi, O, Pant, P, van Teijlingen, E and Wasti, S, 2016. Informed consent in health research: challenges and barriers in lowand middle-income countries with specific reference to Nepal (In press). Developing World Bioethics. (In Press) ] to published in final form at http://onlinelibrary.wiley.com/journal/10.1111/(ISSN)14718847. This article may be used for non-commercial purposes in accordance with Wiley Terms and Conditions for Self-Archiving." 


\title{
INFORMED CONSENT IN HEALTH RESEARCH: CHALLENGES \\ AND BARRIERS IN LOW-AND MIDDLE-INCOME COUNTRIES \\ WITH SPECIFIC REFERENCE TO NEPAL
}

\begin{abstract}
Obtaining 'informed consent' from every individual participant involved in a health research is a mandatory ethical practice. Informed consent is a process whereby potential participants are genuinely informed about their role, risk and rights before they are enrolled in the study. Thus, ethics committees in most countries require 'informed consent form' as part of an ethics application which is reviewed before granting research ethics approval. Despite a significant increase in health research activity in low-and middle-income countries (LMICs) in recent years, only limited work has been done to address ethical concerns. Most ethics committees in LMICs lack the authority and/or the capacity to monitor research in the field. This is important since not all research, particularly in LMICs region, complies with ethical principles, sometimes this is inadvertently or due to a lack of awareness of their importance in assuring proper research governance. With several examples from Nepal, this paper reflects on the steps required to obtain informed consents and highlights some of the major challenges and barriers to seeking informed consent from research participants. At the end of this paper, we also offer some recommendations around how can we can promote and implement optimal informed consent taking process. We believe that paper is useful for
\end{abstract}


researchers and members of ethical review boards in highlighting key issues around informed consent.

Keywords: ethics, research, informed consent, vulnerable population, low-and middle-income countries, Nepal

\section{INTRODUCTION}

The international bioethics guidance on health research was initiated with the Nuremberg Code $^{1}$ and is enshrined in the Declaration of Helsinki (1964) including its most up-to-date revision. ${ }^{2}$ Thus, ensuring full compliance of ethical principles in population-health research is not a new concept. With the growth of health research (both observational and clinical) in low- and middleincome countries (LMICs), associated ethical issues have increasingly becoming a matter of public and scholarly debate. ${ }^{3}$

${ }^{1}$ P. Alderson. Competent children? Minors' consent to health care treatment and research. Soc Sci Med 2007; 65: 2272-2283.

${ }^{2}$ World Medical Association (WMA). 2013. Declaration of Helsinki (last amended by 64th WMA General Assembly, Fortaleza, Brazil, October 2013). Available at: http://www.wma.net/en/30publications/10policies/b3/ [Accesed 12 Dec 2015].

3 A. Zumla \& A. Costello. Ethics of healthcare research in developing countries. J R Soc Med 2002; 95: 275-276; E. R. van Teijlingen \& P.P. Simkhada. Ethical approval in developing countries is not optional. J Med Ethics 2012; 38: 428-430; E.J. Emanuel, D. Wendler, J. Killen \& C. Grady. 
Earlier principles such as those in The Declaration of Helsinki (1964) were dominated by physician-oriented clinical research but were silent on ethical issues related to observational studies in LMICs. Ethical issues raised by health and social care research in LMICs are part of a growing literature. For example, the Nuffield Council on Bioethics in 2002 (The Ethics of Research Related to Healthcare in Developing Countries) has been an important resource for policymakers and researchers. ${ }^{4}$ Research in most LMICs is now overseen by an autonomous body, including Ethical Review Boards (ERBs) or Institutional Review Communities (IRCs). In a LMIC like Nepal with a short history of scientific research, a national body called Nepal Health Research Council (NHRC) established in 1991 approved its first National Ethical Guidelines for Health Research in 2001. Although few health research activities in Nepal started in the early 1950, they were monitored by Government of Nepal through Nepal Medical Research Committee. ${ }^{5}$ At

What makes clinical research in developing countries ethical? The benchmarks of ethical research. J Infec Dis 2004; 189: 930-937; E. van Teijlingen \& P. Simkhada. Failure to apply for ethical approval for health studies in low-income countries. Nepal J Epidemiol 2015; 5: 511-515.

${ }^{4}$ Zumla \& Costello, op. cit. Note 3.

${ }^{5}$ J.R. Sharma, R. Khatri \& I Harper. Understanding Health Research Ethics in Nepal. Dev World Bioeth 2016. 
present, all health-related research conducted in Nepal should be approved by the NHRC or IRC. ${ }^{6}$

Ethics often involve a balance between respecting an individual's liberty/autonomy and the ability to gain benefits for society (i.e. the greater good). Traditionally, specific ethics principles have been formulated based on the four foundation principles: a) respect for autonomy; b) non-maleficence; c) beneficence and; d) justice. ${ }^{7}$ Building on these, Vanclay and colleagues ${ }^{8}$ have recently proposed 18 principles for ethical social research. However, despite the importance of these principles, many researchers in LMICs do not always practise and implement research ethics and principles. Recently, van Teijlingen and Simkhada ${ }^{9}$ highlighted five possible reasons for not applying an ethical approval in low income countries: a) researchers' perceptions that they do not need ethical approval; b) lack of researchers' familiarity with the

${ }^{6}$ Nepal Health Research Council (NHRC). 2011. National Ethical Guidelines
for Health Research in Nepal and Standard Operating Procedures. Kathmandu: Nepal Health Research Council.

${ }^{7}$ T.L. Beauchamp. 2007. The 'four principles' approach to health care ethics. In Principles of Health Care Ethics. R.E Ashcroft, A. Dawson \& J.R. McMillan, eds. Chichester, UK: John Wiley \& Sons: 3-10.

${ }^{8}$ F. Vanclay, J.T. Baines \& C.N. Taylor. Principles for ethical research involving humans: ethical professional practice in impact assessment Part I. Impact Assessment and Project Appraisal 2013; 31: 243-253.

${ }^{9}$ van Teijlingen \& Simkhada, op. cit. Note 3. 
ethics committee; c) researchers applying to the wrong authority; d) time and resource constraints; and e) assumptions that non-clinical research is exempted. In a recent paper, Sharma and colleagues $(2016){ }^{10}$ further argue that research designed to generate evidence for programmatic interventions is not always registered in Nepal, due to the lack of a clear definition as to what is regarded as health research, making it more difficult for ethical bodies to monitor the overall volume of research activities in their country. Perhaps more importantly, when research does not comply with key ethical principles, it may not fully protect the dignity, rights, safety and well-being of its participants. Simultaneously, such work may not assure effective communication with participants which could mean consent decisions being made on poor or even false information. Furthermore, such research can be more difficult to publish in high-quality academic journals which often mandate that the research they published has been granted ethical approval. The International Committee of Medical Journal Editors (ICMJE) guideline, for example, requires ethical approval for any health-related research involving humans. ${ }^{11}$

'Informed consent' is a popular and universal benchmark concept and mechanism for addressing ethical issues in clinical or public health research, and an important element in conducting research ethically. The focus on informed consent falls firmly into the fundamental arena of respect for the

${ }^{10}$ Sharma, Khatri \& Harper, op. cit. Note 5.

${ }^{11} \mathrm{~J}$. Fletcher. Ethical approval for all studies involving human participants.

Can Med Assoc J 2015; 187: 91. 
autonomy of participating individuals, one of the four key ethical principles. ${ }^{12}$ However, informed consent procedures and research ethics are sometimes not given the due attention and care both by the participants and the researchers in LMICs. Riessman (2005) noted that it was difficult to implement informed consent during her study on 'female infertility' in rural villages of India because the participants assume the consent form was a government document. ${ }^{13}$ Moreover even after providing written consent they are not aware of it in a real sense. It is often very difficult to uphold confidentiality during the interview process due to frequent interference of family members. The latter was also noted in focus group studies in rural Nepal, where confidentiality was hard to maintain as it can be culturally and physically difficult to keep non-participants away. ${ }^{14}$

12 I. Harper. Translating ethics: researching public health and medical practices in Nepal. Soc Sci Med 2007; 65: 2235-2247.

${ }^{13}$ C.K. Riessman. Exporting ethics: A narrative about narrative research in South India. Health: 2005; 9: 473-490.

${ }^{14}$ E. van Teijlingen, P. Simkhada \& J. Stephens. Doing focus groups in the health field: some lessons from Nepal. Jhprospect 2013; 12: 15-7. 
Box 1: Informed consent in practice ${ }^{15}$

The researcher has to make sure that the subject's participation is fully informed on:

- what the study is about,

- what its risks and benefits are

- how the results will be used,

- the fact that participation is voluntary and

- can be stopped at any time

- identity will be protected

Informed consent means that all research participants have received adequate information about the research, enabling them to choose freely whether they wish to be involved or not. However, during the field survey such communication between researchers and potential research participants is not always perfect. Consequently, research participants may not always fully understand their role, rights and risks (3R) of taking part in the research. Researchers studying the comprehension of informed consent among clinical trial participants reported that participants both from high- and low- income countries exhibited limited understanding or even misunderstanding about the concept. ${ }^{16}$ This misunderstanding is more frequent in LMICs because local research volunteers are more likely to be illiterate and unaware of ethical principles of research. In this paper, we highlight the challenges in adhering to

\footnotetext{
${ }^{15}$ World Healh Organization. 2015. Global health ethics: key issues. Geneva:
} World Health Organization.

${ }^{16}$ D.W. Fitzgerald, C. Marotte, R.I. Verdier, W.D. Johnson \& J.W. Pape.

Comprehension during informed consent in a less-developed country. Lancet 2002; 360: 1301-1302. 
ethical procedures and taking fully informed consent in LMICs, with examples from Nepal.

\section{VERBAL VERSUS WRITTEN CONSENT: A DILEMMA}

Although some regulations require evidence of consent from the research participants, which in most cases is the signed consent form, these practices however should be adopted carefully. Health research is often conducted with different population groups or subgroups and not everyone is capable of giving written consent. For example, people with limited literacy and young children are unlikely to be able to read the study information sheet and sign the consent form. In the case of a minor (say someone under the age of 12 or in some countries under the age of 16), the use of parental consent is commonly taken as a proxy. Furthermore, a lack of clear guidance in most LMICs on the consent taking procedure poses further challenges to being able to adherence to ethics principles. One example is that despite stating clearly around importance of informed consent and its process to ensure it is voluntary, the NHRC/IRC guideline ${ }^{17}$ for Nepal is silent about verbal or written consenting methods raising ambiguity in the process. The context in which verbal or written consent is appropriate should be clearly informed by NHRC guidance.

Researchers working with vulnerable or socially disadvantaged groups or those with lower literacy may ask potential participants to write their name or signatures. However, such public record may in practice result in a breach of

\footnotetext{
${ }^{17}$ Nepal Health Research Council, op. cit. Note 6.
} 
confidentiality. Use of thumb prints for illiterate participants as an alternative also has certain disadvantages such as some studies have reported that the participants may think this may have legal implications ${ }^{18}$ or that they could be deceived, which may in turn develop mistrust about the study. In such situations where written consent is not possible and plausible, witnessed consent from an independent individual would be a better alternative. The reason for undertaking witnessed consent should be recorded and the person seeking the consent must be able to show ethical considerations.

Ideally, a 'participant information sheet' must be provided to all research participants in advance offering sufficient time to consider the decision regarding their participation before requesting formal consent. The information sheet should be provided in the participant's local language or in the language the participant is most comfortable with. The information sheet should clearly explain the key aspects of the research: background to the study, aim and objectives, potential participants of the study, possible risks and benefits of the research or experiments (both direct and indirect), incentives, risks and benefits of other options (including not getting treatment), opportunity to ask questions, information that would enable participants to make a decision, contacts details of the researchers including ethical approval boards and right to refuse from the participation and

${ }^{18}$ P. A. Marshall. 2007. Ethical Challanges in Study Design and Informed Consent for Health Research in Resource-Poor Setting. Geneva: World Health Organization. 
withdrawal from the research at any time (i.e. complying with the Declaration of Helsinki $\left.{ }^{19}\right)$. Researchers' skills are particularly important in communicating the importance of the research and describing its aims and objectives, including the potential risk and benefit to the research participants. This necessitates that research capacity building activities (e.g. training, workshops), that to date have mostly focused on familiarization of research tools or data collection approach, should offer experiential learning approaches to strengthen researchers' skills.

\section{LOCAL LANGUAGE IN A RESEARCH: OPPORTUNITY OR}

\section{BARRIER?}

As health sector research or services delivery in LMICs are heavily dependent on external funding ${ }^{20}$, much of their research is likely to be collaborative in nature where English language (or any other former colonial language) is commonly used between research implementing partners and funding agencies. It is a common practice for researchers from LMICs to draft research protocols, tools used such as questionnaires and consent forms in English. Where documents are initially prepared in a language other than that of the research participants, those documents should be translated into the given local (vernacular) language and validated. The latter can be done by doing back translation into English by an independent translator in order to make sure the original meaning is not lost during the translation phase. There may

\footnotetext{
${ }^{19}$ World Medical Association, op. cit. Note 2

${ }^{20}$ Sharma, Khatri \& Harper, op. cit. Note 5.
} 
be financial and time implications validating such translations, ${ }^{21}$ but providing information about the research in participants' preferred languages may have a multiple benefits including free expression. ${ }^{22}$ For example in countries as diverse as Nepal, local languages are often preferred in terms of both culture and linguistics. However, it is not always easy to carry out research among some ethic groups or indigenous populations (IP) as their traditional culture or language can be barriers. Limited literature on IP reflects that IP have often little or no representation or rights with respect to the research process, or the interpretation and use of the resulting data. Therefore, research needs of IP and ethics groups should be explored, after such exploration research should be organized, designed and conducted in a manner that takes account of cultural differences. ${ }^{23}$

\section{LEGAL AGE TO CONSENT}

The threshold of the age of consent is another contentious area. Alderson (2007) argues that research which could equally well be done with adults should never be undertaken with children, ${ }^{24}$ but this is not always possible.

${ }^{21}$ H.J. Smith, J. Chen \& X. Liu. Language and rigour in qualitative research: Problems and principles in analyzing data collected in Mandarin. BMC Med Res Methodol 2008; 8: 1-8.

${ }^{22}$ K. Kumar. 2006. Conducting Mini Surveys in Developing Countries. USA:

Bureau for Policy and Program Coordination, USAID.

${ }^{23}$ Vanclay, Baines \& Taylor, op. cit. Note 8.

${ }^{24}$ Alderson, op. cit. Note 1. 
The statutory age of consent to treatment or being involved in a research varies considerably between countries from 12 to $19 .^{25}$ Although consent involves evaluating risks and considering whether to undertake them, for selfevident practical reasons research participants such as children may not understand the risk involved in taking part in a research. There could be extra risks as children tend to be more vulnerable than adults for being harmed in the short or long term by interventions. ${ }^{26}$ Children are generally less likely to resist and often more likely to respond to researchers' requests. In Nepal, for example, there is a practice of taking guardians' or parents' consent if research involves children. A clear threshold age of consent has not been recommended in NHRC guidelines. However, we have observed that most of the researchers in Nepal consider age 16 years and above as a threshold to solicit informed consent as it is a legal age to obtain Nepalese citizenship and to enroll in a voter roll. This practice based on legal standard is inconsistent with the evidence from other settings which showed that participants aged 14 years or more have an equal competency to provide informed consent as in the adults. ${ }^{27}$ Thus, it would be useful to explore the relationship between age and

${ }^{25}$ Ibid.

26 Ibid.

${ }^{27}$ L. A. Weithorn. Children's capacities to decide about participation in research. IRB 1983;5:1-5; E.J. Susman, L.D. Dorn \& J.C. Fletcher. Participation in biomedical research: the consent process as viewed by children, adolescents, young adults, and physicians. J. Pediatr 1992;121: 547552. 
competency among children and minors in Nepal based on key capabilities required to provide informed consent such as to what extent they understand research, assess risks and benefits, and possess decision making skills. This would also be in line with the Article 12 of the 'United Nations Conventions on the Right of the Child' which clearly states that child's participation in the decision making process should be appropriate to their level of maturity. ${ }^{28} \mathrm{We}$, therefore, recommend NHRC, the research governing body of Nepal, to act on determining evidence based threshold age of consent. Although parents or guardians with legal responsibility should be consented for the children incompetent to consent, children themselves should also be asked for their consent appropriately and in a transparent way with witnesses and records of the consent taking process should be kept. It is advisable to inform young children about the research and/or interventions before obtaining their consent by preparing and sharing imaginative leaflets, videos or explanatory toys. However, in the context of research among the most at-risk populations such as sex workers, or drug misusers, there is a greater chance of such childparticipants being discriminated against or even rejected by their families,

${ }^{28}$ The Office of the United Nations High Commissioner for Human Rights (OHCHR).1989.Convention on the Right of the Child. Geneva: OHCHR. Available at: http://www.ohchr.org/en/professionalinterest/pages/crc.aspx [Accessed 2 July 2016] 
particularly in LMICs if the research makes their status as a sex worker or drug user common knowledge.

\section{INFLUENCE ON DECISION MAKING}

In most LMICs, the response rates are often quite high, in some cases up to $100 \%$. High response rate could be due to a number of reasons, such as (i) there is very little research taking place in LMIC, especially in rural areas, therefore participants might get involved due to their curiosity or they may even be pleased to be invited to participate; (ii) direct or indirect involvement of local health care workers or community health volunteers (such as female community health volunteers in Nepal), who have a very influential role in the diagnosis/treatment of common non-serious diseases in the locality. Support from locally well-regarded individuals such as the elders, teachers and community leaders might also contribute in high response rates. There is also a notion that in LMICs, researchers are generally of higher socio-economic status and this socio-economic inequality leads the participants to feel that they are obliged to participate in a study when requested..$^{29}$ In clinical research, some individuals participate in the research with the aim of getting their health checked for free as they cannot afford expensive medical screening. It may be also possible that they take part in interventional studies because the treatments are provided free of cost. Some researchers provide financial incentives or compensate for the time of participants to increase the response

${ }^{29}$ S.R. Benatar. Reflections and recommendations on research ethics in developing countries. Soc Sci Med 2002; 54: 1131-1141. 
rates. Equally, it may be true that participants develop a relationship of trust with researchers and they participate in order to help the research to move forward but these could be limited to those participants with a higher educational status.

As in many LMICs, health research in Nepal has increased drastically in the last few decades. One of the most important research ethics principles is 'autonomy' and gender is often an important determinant of such autonomous decision-making processes. Acharya and colleagues ${ }^{30}$ highlighted that women, particularly the uneducated women in Nepal, have less power and autonomy in household level decision-making compared to men. The practices where spousal permission and company are needed in order to visit a health clinic for a diagnosis or treatments have been documented. ${ }^{31}$ In such situations where women require a permission from their husbands or other family members to be allowed to participate in health research. This raises the question; to what extent are women (a) informed and able to offer informed consent; and (b) whether they can culturally choose not to consent once their husband has given permission for her research participation. Currently, research on

${ }^{30}$ D.R. Acharya, J.S. Bell, P. Simkhada, E.R. van Teijlingen \& P.R. Regmi. Women's autonomy in household decision-making: a demographic study in Nepal. Reprod Health 2010; 7: 15.

${ }^{31}$ B. Gyawali, J.J. Keeling, E. van Teijlingen, L. Dhakal \& A.R. Aro. Cervical cancer screening in Nepal: Ethical considerations. Medicoleg Bioeth 2015: 16. 
autonomy or the decision-making power of different genders in relation to their participation in a health research is lacking. Other possible biases in decision-making may arise when a health facility/hospital or health professionals are themselves directly involved in the research. It is the duty of researchers to convey to the participants by means of the informed consent that the decision on whether to participate or not will not have any consequence for their ongoing or future service from the health care facility, and that they are free to withdraw from the research at any time and without providing any reason whatsoever. Moazam and Jafarey ${ }^{32}$ opined that socially and culturally entrenched norms and Islamic laws have a greater influence on bioethics in Pakistan.

\section{RISKS AND BENEFITS}

The primary aim of the health research is to benefit the population or its subsection either immediately or in the future and general guiding principle is that the benefits should always outweigh the risks. Some participants might ask 'what beneficial outcome am I going to get if I take part in this research?' The researcher should be able to apprise the participants on the benefit that their participation may confer in the future in due course. In Nepal, providing the findings of the research to a local health authority such as a district health office would demonstrate benefit to the participating community. As an immediate benefit, we do not recommend any monetary incentive to the

\footnotetext{
${ }^{32}$ F. Moazam \& A.M. Jafarey. Pakistan and biomedical ethics: Report from a Muslim country. Camb Q Healthc Ethics 2005; 14: 249-255.
} 
participants but where appropriate, people should be reimbursed for their travel costs. However, providing other forms of incentive related to the research area, e.g. providing dietary supplements in research on maternal and child health or condoms to sexually active populations, could be acceptable.

The issue of potential risks particularly involving biological specimens (e.g. blood, sputum and urine) and the use of medical devices are important and to be discussed in advance, a clear plan to minimize the risks should be listed. Participants who suffer injuries during the research, even minor ones (i.e. finger-pricked for blood sample) should be dealt with caution and a log of all accidents should be kept up to date. The participants should be informed in advance on any potential risk such as inflammation or pain. Despite a very rare probability of potential risk, participants should be clearly informed about it a priori. Potential risks need to be fully described (e.g. in the research information sheet, consent form) and discussed with all potential study participants prior to participation. It should clearly mention the contact person and reporting procedures in case of any social or physical harm that may arise as a result of taking part in the study. Research staff should be aware and trained with appropriate counselling skills to refer such participants to services within their communities. An account of potential social harm outcomes resulted from the participation during research also need to be appropriately reported to all relevant ERBs and IRCs. 
In addition, psycho-social risks (e.g. emotional responses to a research topic) may occur to participants in social studies or research in sensitive issues. ${ }^{33}$ There are also good reasons not to underestimate the importance of emotional and social risks. If researchers feel some questions may cause psycho-social risk to the participants due to individual circumstances, it would be beneficial to discuss a priori with local research staffs. This is the responsibility of the researcher to describe eloquently and in unambiguous ways, their protocol and in the script of the consent form ensuring whether it is done or not, may fall under the remit of the ERBs and peer reviewers that should be mentioned in the guidelines. If an application fails to address the issues, the reviewers should ask the researchers to address the questions clearly.

\section{A WAY FORWARD}

Research participants from LMICs such as in Nepal, can comprehend a consent-taking process if sufficient information is provided by the researchers in demonstrably clear and easy to understand ways. We suggest that ways of informing children, populations at risk and indigenous people, respecting their autonomy and their vulnerability, to help to fulfil the longstanding guidance that 'research involving these vulnerable populations is important for the benefit of participants'. Incorporating 'ethics training' in postgraduate and public health training programs would help to explain the importance and

${ }^{33}$ M.M. Burgess. Proposing modesty for informed consent. Soc Sci Med 2007; 65: 2284-2295. 
maintain the adherence fully informed consent and ethics in research. The following suggestions are therefore made to indicate ways forward:

- Researchers must ensure that the informed consent-taking process is conducted appropriately and detailed information about risks and benefits are provided to participants in easily understood ways.

- Having participated in the research and assumed risks, the participants and host community have a right to know what was found and its implications for public health and social-care policies. Publishing in Open Access journals which are available free of cost to read on the web, increases the chance that someone in LMICs can read your research findings, but English language would still be a barrier, particularly to those living in the rural communities.

- Ethical review boards should ensure all research subjects are at minimum or low risk. ERBs' responsibilities include protecting potential research participants from undue pressure or coercion that could be used on them to take part in a study and play a key role in preventing distortion of recruitment, particularly by imposing tight restrictions on how participants may be approached.

- Health research councils should act to determine evidence based threshold age of consent and discourage current practice of considering legal standard.

- Research funders and collaborators should ensure that research in host countries is conducted in compliance with the requirements laid out by local ERBs. 
Finally, we have a notion that only properly conducted consent process respects the autonomy and fundamental human rights of a study participant, including freedom from explicit or implicit exploitation. It also protects researchers from criticisms, complaints, mitigation; and participants from any risk during the recruitment and participation phases. This paper is hopefully of use to researchers in LMICs as well as members of ethical review boards in highlighting key issues around informed consent.

\section{CONFLICT OF INTERESTS}

The authors declared no potential conflicts of interest with respect to the research, authorship, and/or publication of this article.

\section{FUNDING}

The author received no financial support for the research, authorship, and/or publication of this article.

\section{Biographies}

Pramod R. Regmi, Public Health Researcher and Research Fellow at Bournemouth University, UK. His research interests are young people's sex and relationships, diabetes, health promotion, ethics in population health research. He holds an MA degree in Demography, MSc (Health Services and Public Health Research) and PhD (Public Health). He previously worked for FHI360 (Nepal) as a Senior Surveillance and Research Specialist. 
Nirmal Aryal holds an MSc degree in Public Health from UK and is currently a $\mathrm{PhD}$ candidate at the University of Otago, New Zealand. His research interest includes epidemiology of cardiovascular diseases and related risk factors.

Om Kurmi, Senior Scientist in Respiratory Epidemiology, Clinical Trial Service Unit and Epidemiological Studies Unit (CTSU), Nuffield Department of Population Health, University of Oxford, UK. His main research interest is environmental epidemiology of chronic diseases, particularly cardiorespiratory health in low-and middle-income countries related to air pollution (both ambient and household air pollution), occupational exposures and smoking (active and second-hand smoking).

Puspa Raj Pant is a researcher at the University of the West of England, UK. His recent work is more on injury prevention and safety promotion in Nepal where he has used a range of approaches to achieve research objectives-from mixed-methods to community mobilisation and advocacy.

Edwin van Teijlingen, Professor of Reproductive Health Research at Bournemouth University, UK. He has over 220 peer-reviewed publications in the field of public health, social sciences and the organisation of health care systems and more than sixty of his publications focus on Nepal. He is coeditor of the edited volume The Dynamics of Health in Nepal (Social Science Baha/ Himal Books, Nepal).

Sharada P. Wasti, Research Coordinator, Institute for Reproductive Health, Georgetown University, USA (Nepal based staff). He has worked in the field of public health for over a decade in Nepal. He holds a Master's degree in Health Care Management and in Population Studies from Nepal and an MSc 
in International Health and a PhD (Public Health) from the UK. He is lead editor of the edited volume The Dynamics of Health in Nepal (Social Science Baha/Himal Books, Nepal). 